\title{
Entwicklung und Überprüfung von Erhebungsinstrumenten zur Erfassung der Fachkompetenz (angehender) frühpädagogischer Fachkräfte
}

\author{
Janina Limberger ${ }^{1}$, Annika Lorenzen ${ }^{1}$, Charlotta Wirth ${ }^{1}$, Janina Strohmer ${ }^{2}$ \\ und Klaus Fröhlich-Gildhoff'
}

'Zentrum für Kinder- und Jugendforschung (ZfKJ) an der Evangelischen Hochschule Freiburg

${ }^{2}$ Pädagogische Hochschule Freiburg

\begin{abstract}
Zusammenfassung: Die Akademisierung des kindheitspädagogischen Sektors, die damit einhergehenden Professionalisierungsansprüche und die zunehmende Fokussierung auf die Kompetenzorientierung im Aus- und Weiterbildungsbereich bilden den Grundstein für das gestiegene Interesse an Möglichkeiten zur Erfassung von Fachkompetenzen im Bereich der Kindheitspädagogik. Nachfolgend werden die Entwicklung sowie die psychometrische Überprüfung dreier entsprechender Erhebungsinstrumente beschrieben. Von Mai 2017 bis April 2020 wurden hierfür sowohl das theoretische Wissen als auch das Handlungswissen im frühpädagogischen Bereich von 831 angehenden und geprüften pädagogischen Fachkräften (Fachschüler_innen in der Ausbildung zur/m Erzieher_in, Bachelor-Studierende der Kindheitspädagogik, pädagogische Fachkräfte in Kindertageseinrichtungen) erfasst. Eine explorative Faktorenanalyse ergab eine dreidimensionale Struktur der erfragten Kompetenzbereiche, wenngleich aufgrund schwacher Reliabilitäten beim Handlungswissen übergeordnete Faktoren gebildet wurden. Auf Basis der Ergebnisse einer Interkorrelationsanalyse konnte zusätzlich gezeigt werden, dass die Erhebungsinstrumente eine gute konvergente Validität aufweisen. Dies deutet darauf hin, dass die Erfassung von theoretischem Wissen und Handlungswissen zur Einschätzung der Fachkompetenz sinnvoll erscheint. Insgesamt können die Erhebungsinstrumente nach erster psychometrischer Überprüfung als akzeptable mögliche Herangehensweisen zur Kompetenzerfassung eingeschätzt werden.
\end{abstract}

Schlüsselwörter: Instrumentenentwicklung, Kompetenzerfassung, Fachkompetenzen, psychometrische Überprüfung, Kindheitspädagogik, pädagogische Fachkräfte

\section{Development and Verification of Instruments for Acquisition of Professional Competence of (Prospective) Early Childhood Educators}

Abstract: The academization of the early childhood field, the aspiration of professionalization and the enhanced competence-focus in professional and further training provide the basis for enhanced interests in the assessment of competencies in early childhood education. In this article, the development and psychometric validation of three assessment-tools are described. Therefore, from 05/ 2017 till $04 / 2020$ we gathered theoretical and action knowledge of overall 831 educational professionals (early childhood trainees, early childhood students, approved early childhood educators). In this article, three assessment-tools for assessing theoretical knowledge as well as action knowledge are described, regarding contentual and psychometric aspects. The exploratory factor analysis revealed a three-factorial structure of the areas of competence, although, superordinate factors were formed due to weak reliabilities in the action knowledge. Based on intercorrelation analysis, the instruments also show convergent validity, suggesting that the gathering of theoretical knowledge and action knowledge is useful for assessing professional competencies. Overall, due to psychometric testing the survey instruments can be assessed as acceptable possible approaches for acquisition of professional competence.

Keywords: instrument development, competence assessment, professional competence, early education, early childhood educators 


\section{Thematische Hinführung und aktueller Forschungsstand}

Die zunehmende Fokussierung der Kompetenzorientierung im Aus- und Weiterbildungsbereich und der damit verbundenen Lernergebnisse (z. B. Drieschner, 2009) hat im Zuge der Akademisierung und damit einhergehenden Professionalisierungsansprüche (Mischo \& FröhlichGildhoff, 2011) Einzug in die Kindheitspädagogik gehalten. Vor diesem Hintergrund besteht ein großes Interesse an Möglichkeiten, verschiedene Kompetenzbereiche zu erfassen. Bisherige Bemühungen zeigen in der Zusammenfassung, dass der Bereich der Lehrer_innenforschung eine Vorreiterrolle einnahm und seit einigen Jahren bereits Ergebnisse vorliegen (siehe Frey, 2006; Hohenstein, Kleickmann, Zimmermann, Köller \& Möller, 2017; Neuweg, 2015, Witteck, Schneider \& Kramer, 2020). Während sich etliche bisherige Forschungen (Klaudy, Schütz \& Stöbe-Blossey, 2014; Kuckarz, Mackowiak, Ziroli, Kauertz, Rathgeb-Schnierer \& Dieck, 2014) im frühpädagogischen Sektor in Professionalisierungszusammenhängen mehr mit den Grundlagen der generell benötigten Fachkompetenzen befassten als mit deren expliziten Erfassung, startete 2015 eine breit angelegte Initiative des Bundes zur Erfassung von Fachkompetenzen im Hochschulsektor („KoKoHS - Kompetenzmodellierung und Instrumente zur Kompetenzerfassung im Hochschulsektor"; BMBF, 2020). Im Bereich der frühpädagogischen Fachkräfte in der Praxis gab es in der Vergangenheit einige Vorhaben zur Fachkompetenzmessung: So untersuchten Verbeek und Perels (2018) überfachliche berufsrelevante Kompetenzen anhand eines Validierungsverfahrens bei angehenden Erzieher_innen (siehe Verbeek, 2016); das Projekt AVE (Ausbildung und Verlauf von Erzieherinnen-Merkmalen; Mischo, 2017) befasste sich mit der Erfassung der Kompetenzen im Bereich der Sprache und das Forschungsprojekt „ProKOMma“ (BMBF, 2020) mit dem mathematischen Fachwissen angehender pädagogischer Fachkräfte. Darüber hinaus explizierten Granzow (2017), anhand eines Struktur- und Messmodell zur Erfassung beruflicher Kompetenzen für das Praxisfeld der Kindertagesstätte, sowie Blömeke et al. (2015), zu Leistungstests zur Messung der professionellen Kompetenzen frühpädagogischer Fachkräfte, durch ihre Publikationen die Vielfalt an Messmöglichkeiten (siehe Kuckarz et al., 2014). Zusammengefasst lässt sich jedoch ein Desiderat hinsichtlich vergleichender Fachkompetenzerfassung sowohl bei angehenden pädagogischen Fachkräften (Hochschüler_innen, Fachschüler_innen), als auch bei bereits ausgebildeten Fachkräften im Bereich der Kindheitspädagogik feststellen - dies gilt vor allem hinsichtlich der Erfassung verschie- dener Wissensbereiche. Insbesondere die Kombination aus der Erfragung von Theorie- und Handlungswissen sowie der Vergleich von Fachschüler_innen und Hochschüler_innen in Ausbildung und frühpädagogischen Fachkräften in der Praxis, stellt verglichen zu den rezipierten Ansätzen vergleichbarer Studien, einen umfänglichen und neuen Ansatz dar, weshalb nachfolgend die Entwicklung und Überprüfung von drei Instrumenten zur Erfassung der fachlichen Kompetenzen von (angehenden) frühpädagogischen Fachkräften vorgestellt wird.

\section{Methodisches Vorgehen}

\section{Studiendesign \& Stichprobe}

Die Instrumente wurden im Rahmen des Forschungsprojektes „Individuelle kompetenzorientierte Feedbacks als Methode der Professionalisierungsbegleitung frühpädagogischer Fachkräfte (InKoFeed) " an der Evangelischen Hochschule in Freiburg entwickelt, welches im Warte-Kontrollgruppendesign einerseits sowohl das Ziel der empirischen Validierung bestehender frühpädagogischer Kompetenzmodelle, andererseits die Überprüfung der Wirksamkeit und Wirkung der individuellen Kompetenz-Rückmeldungen in verschiedenen Aus- und Weiterbildungssettings verfolgt. Für die psychometrische Überprüfung der entwickelten Erhebungsinstrumente wurde der erste Messzeitpunkt des Projekts verwendet. Insgesamt füllten in der Zeit von November 2017 bis April 2018 831 Teilnehmende aus dem Bundesgebiet den Fragebogen zur Erfassung des theoretischen Wissens (FTW) aus (Alter: $M=23.30$ Jahre, $S D=7.95,90,0 \%$ weiblich), davon 326 Hochschüler_innen (39,2\%), 366 Fachschüler_innen $(44,0 \%)$ und 139 pädagogisch tätige Fachkräfte aus Kindertageseinrichtungen (16,7\%). Die Stichprobe der Instrumente zur Erfassung des Handlungswissens ( $F H W$, $D H W$ ) umfasst 418 Teilnehmer_innen (Alter: $M=23.76$ Jahre, $S D=8.68,93,3 \%$ weiblich; 50,3\% der Gesamtstichprobe des $F T W$ ), aufgeteilt auf 170 Hochschüler_innen (40,7\%), 165 Fachschüler_innen (39,5\%) und 83 pädagogisch tätige Fachkräfte (19,9\%).

Der FTW wurde bundesweit an sieben Hochschulen (t1, 3. Semester), acht Fachschulen (t1, Unterkurs) landesweit in Baden-Württemberg und in 21 Kitas in der Region Freiburg als Paper-Pencil-Variante eingesetzt. Sowohl die Fallvignetten, als auch die Dilemmasituationen wurden als Online-Version angelegt und konnten mithilfe eines Links, welcher bei der Vor-Ort-Befragung ausgehändigt wurde, von allen Teilnehmer_innen, die zuvor den FTW ausgefüllt hatten, anschließend ausgefüllt werden. 


\section{Theoretischer und konzeptueller Hintergrund der Erhebungsinstrumente}

Theoretische Grundlage für die Erfassung von Fachkompetenz im Rahmen der vorliegenden Instrumentenentwicklung bildet das vielfach rezipierte Kompetenzmodell von Fröhlich-Gildhoff et al. (2014). Das professionelle Wissen, welches nach den Autor_innen aus wissenschaftlich-theoretischem Wissen und implizitem Erfahrungswissen besteht, spielt laut Modell bei der entstehenden Handlungsplanung und -bereitschaft eine große Rolle ebenso wie die Situationswahrnehmung und -analyse. Insbesondere die Erfragung des Wissens (Theorie- als auch Handlungswissen) steht im Fokus der hier referierten Forschung. Dieses Modell bezieht sich auch auf Weinert (2001).

Die Erfassung und Einteilung in einzelne Themenkomplexe, welche innerhalb der Messinstrumente erfolgte, entstammt dem Orientierungsrahmen für die Hochschulausbildung von Kindheitspädagog_innen (Robert Bosch Stiftung, 2008), der später auch für Fachschüler innen angeglichen wurde (Autorengruppe Fachschulwesen, 2011).

Der Qualifikationsrahmen enthält hierbei eine inhaltlich-thematische Einteilung in fünf unterschiedliche Kompetenzbereiche: 1. Kind in Beziehung zu sich und anderen, 2. Kind und Welt, 3. Eltern und Bezugspersonen, 4. Institution und Team sowie 5. Netzwerke, welche auch in dieser Form zunächst übernommen wurde. Für eine ganzheitliche Erfassung akademischer Kompetenzen wurde zudem ein sechstes Themengebiet nach dem erweiterten Orientierungsrahmen für Hochschulen (6. Forschung, Recherche und Evaluation) bei der Instrumentenentwicklung hinzugefügt (Robert Bosch Stiftung, 2008). Der Qualifikationsrahmen umfasst hierbei im Wesentlichen diejenigen Inhaltsbereiche, die sich in einem Großteil der Kernarbeitsfelder (Bildung, Betreuung und Erziehung von Kindern im Vorschulalter) wiederfinden. Wenngleich er damit weitere wesentliche Inhaltsbereiche wie beispielswiese Diversität und Inklusion weitestgehend ausspart, so stellt er doch ein Rahmenmodell dar, welches sowohl auf akademischem als auch auf nicht-akademischem Niveau Konsens und Anwendung findet sowie bereits mehrfach als Grundlage für weitere Untersuchungen herangezogen wurde (Strohmer \& Mischo, 2016).

\section{Entwicklung der Erhebungsinstrumente}

Fragebogen zur Erfassung des theoretischen Wissens (FTW): Zur Erfassung deklarativer Wissensbestände im Bereich der Kindheitspädagogik wurde ein standardisierter Fragebogen entwickelt. Dieser umfasste insgesamt 66 MultipleChoice-Fragen zu den sechs pädagogischen Kompetenz- bereichen (pro Kompetenzbereich 11 Fragen, s. Kasten 1). Die Entwicklung der Items für den Fragebogen orientierte sich zum einen an bestehenden Instrumenten (u.a. aus den Studien von Fröhlich-Gildhoff \& Böttinger, 2018; Fröhlich-Gildhoff et al., 2014; Mischo et al., 2016). Zum anderen wurden neue Items anhand des Orientierungsrahmens für Hoch- und Fachschulen (Autorengruppe Fachschulwesen, 2011; Robert Bosch Stiftung, 2008) und weiterer einschlägiger Fachliteratur konstruiert.

Kasten 1. Beispielitems aus dem Kompetenzbereich 2 „Kind und Welt“

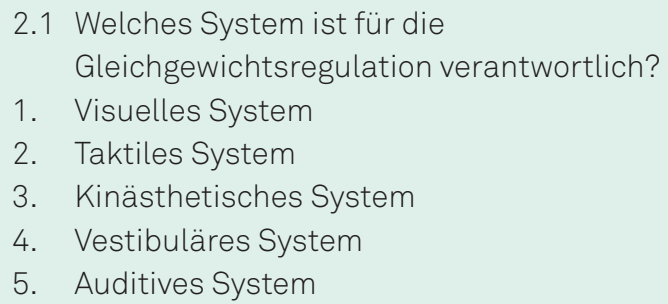

2.9 Was bedeutet „Semantische Kompetenz“

1. Sprachlaute erkennen, unterscheiden und produzieren zu können.

2. die Bedeutung von Wörtern und ihre Kombination zu kennen.

3. Intonation, Betonung, rhythmische Gliederung und Satzmelodie erkennen und richtig anwenden zu können.

4. Sätze sozial und kommunikativ angemessen verwenden und Dialoge strukturieren.

Fallvignetten zur Erfassung des Handlungswissens (FHW): Um die Fachkompetenzen der Teilnehmer_innen hinsichtlich des beruflichen Handelns und der Planung der eigenen Handlung zu erfassen, wurde für jeden der sechs pädagogischen Kompetenzbereiche eine eigene standardisierte Fallvignette (Bearbeitung von Fallbeispielen aus dem pädagogischen Alltag in Kindertageseinrichtungen) eingesetzt. Bei den Fallvignetten wurden ebenfalls sowohl bereits bestehende Instrumente (u.a. aus der Studie von Fröhlich-Gildhoff, Rönnau-Böse \& Tinius, 2017) verwendet, als auch - orientiert an der Fachliteratur - eigene Fallvignetten entwickelt (s. Kasten 2). Über die zusätzliche Erfassung mittels Vignetten, in denen die eigenen kurzund längerfristigen Handlungsideen formuliert werden sollen, soll eine weniger theorie- und stärker handlungsorientierte Fokussierung erreicht werden. In der Beschreibung von konkreten Handlungen spiegeln sich vermutlich in stärkerem Maße auch implizite und prozedurale Wissensbestände, die unter Umständen fachlich nicht exakt benannt werden können (Barter \& Renold, 1999), wider als in der Beantwortung theoretischer Fragen. 
Kasten 2. Beispiel zum Kompetenzbereich 2 „Kind und Welt“

In der Kita-Gruppe schauen Sie sich mit Max (4,0 Jahre) und Tina (4,5 Jahre) gemeinsam ein Wimmelbuch an. In dem Wimmelbuch ist ein Haus abgebildet, in das man hineinsehen kann. Es sind verschiedene Zimmer mit unterschiedlichen Ausstattungen abgebildet. In einem Zimmer sitzt eine Person auf der Toilette. Seit zwanzig Minuten unterhalten sich Max und Tina über den Toilettengang und die Person, die auf der Toilette sitzt. Max sagt: „Hihihi, der sitzt auf dem Klo. Der macht bestimmt AA." und Tina fragt Sie selbst daraufhin: „Hast du heute auch schon AA gemacht?". Beide Kinder kichern laut.

1. Was würden Sie in dieser Situation tun / zu den Kindern sagen?

2. Wie würden Sie ggf. langfristig das Thema der Kinder aufgreifen?

3. Wie begründen Sie Ihr konkretes Handeln? Welche handlungsrelevanten Aspekte nehmen Sie in der Situation wahr?

Dilemmasituation zur Erfassung des Handlungswissens (DHW): Inhalt der Methode der standardisierten Dilemmasituationen ist die Beschreibung einer komplexen bzw. uneindeutigen und herausfordernden Situation im pädagogischen Alltag einer Kindertageseinrichtung, welche bei der Bearbeitung verschiedene anwendungsbezogene Wissens- und Reflexionsprozesse der Teilnehmer_innen herausfordern. Mit der Methode ist es somit möglich, die Einschätzung von und Reaktion auf spezifische kritische Situationen und damit die Fähigkeit zur Situationswahrnehmung und Situationsanalyse der Teilnehmer_in- nen zu erfassen. Die Dilemmasituationen wurden zunächst für alle Inhaltsbereiche entworfen, aus forschungspragmatischen Gründen (Verständlichkeit, Interraterreliabilität und Länge der Testung) jedoch nur für die Handlungsfelder 1-4 ausgearbeitet - im Endeffekt wurde jeder Personaus Zeitgründen nur eine Dilemmasituation mittels zufälliger Zuweisung vorgelegt. Die Dilemmasituationen wurden ebenfalls sowohl aus bestehenden Studien entnommen (u.a. Fröhlich-Gildhoff, Pietsch, Wünsche \& Rönnau-Böse, 2011; Fröhlich-Gildhoff et al., 2017; Weltzien \& Lorenzen, 2016), als auch anhand von Fachliteratur entwickelt (s. Kasten 3).

Alle drei entwickelten Erhebungsinstrumente wurden einem mehrschrittigen Pretest-Verfahren unterzogen: Zuerst wurde eine fachliche Absicherung der in einer ersten Runde entwickelten Items aller drei Erhebungsinstrumente durch Expert_innen (Wissenschaftler_innen anderer Hochschulen, Leitung Kindertageseinrichtungen, Alumni Master der Kindheitspädagogik) vorgenommen. Dazu wurde eine Expert_innenbefragung einerseits über einen Expert_innentag (Wissenschaftler_innen von Hochschulen aus Deutschland und Österreich, dem Deutschen Jugendinstitut [DJI] sowie städtischen Trägern von Kindertageseinrichtungen) (Diskussion) und eine Online-Expert innenrunde andererseits (Kommentierung) durchgeführt. Des Weiteren wurde mithilfe der „Methode des Lauten Denkens“ (Hunkirchen, 2005; Konrad, 2010) die Benutzerfreundlichkeit der drei Erhebungsinstrumente überprüft. Der FTW wurde als einziges Instrument im Rahmen einer Präsenzerhebung durchgeführt, daher wurde dieser zusätzlich einer Überprüfung unter realistischen Bedingungen (u.a. zur Ermittlung der anzusetzenden Zeitdauer; $N=53$ ) unterzogen. Anhand der Erkenntnisse aus allen Vorgehensweisen

Kasten 3. Beispiel zum Kompetenzbereich 2 „Kind und Welt“

Alexander (5 Jahre) beschäftigt sich mit dem Thema „Raketen“, ist sehr begeistert davon und erzählt in der Gruppe nur noch von diesem Thema. Die pädagogische Fachkraft möchte mit Alexander darüber ins Gespräch kommen und beginnt eine Unterhaltung mit dem Jungen. Da sich die pädagogische Fachkraft nur sehr wenig mit „Raketen“ auskennt, wird sie bei der Unterhaltung in ihren Aussagen und Fragen fortlaufend von Alexander korrigiert, der gut über Raketen Bescheid weiß. Die Fachkraft fühlt sich durch die Korrektur des Jungen zunehmend überfordert und wendet sich mehr und mehr dem Mädchen Lisa (3,6 Jahre) zu, welches ruhig neben ihnen spielt. Lisa hat am Morgen bei der Verabschiedung ihrer Mutter sehr viel geweint und sich erst vor zehn Minuten wieder richtig beruhigt. Alexanders Versuche sich weiter mit der Fachkraft über Raketen zu unterhalten, erwidert diese mit Hinweisen auf das „Mutter, Vater, Kind-Spiel“ von Lisa, in dem er ja mitspielen und die Rolle des Vaters als Astronaut übernehmen könne. Richtig zufrieden ist die pädagogische Fachkraft mit der Situation nicht.

1. Worin besteht in Ihren Augen der Kern des Dilemmas in dieser Situation?

2. Wie erklären Sie sich die Handlungsweisen und Reaktionen der einzelnen Akteure und wie bewerten Sie diese?

3. Wie würden Sie als verantwortliche Fachkraft agieren und wie begründen Sie Ihr (fiktives) Handeln? Auf welche Theorien und Erfahrungen stützen Sie Ihre Entscheidung?

4. Welche Fragen / Herausforderungen ergeben sich für Sie persönlich und für Ihre eigene fachliche Entwicklung aus dieser Situation? 
wurden die Erhebungsinstrumente weiter angepasst und finalisiert.

\section{Ergebnisse der psychometrischen Überprüfung}

\section{Fragebogen zur Erfassung des theoretischen Wissens (FTW)}

Die Überprüfung der Dimensionalität des theoretischen Fragebogens $(F T W)$, welche der Klärung der Frage dient, ob die entwickelten Items die sechs Handlungsfelder der Kindheitspädagogik widerspiegeln, wurde mittels einer explorativen Faktorenanalyse für dichotome Daten mit Mplus (Muthen \& Muthen, 2010) durchgeführt. Die Analyse wies auf eine dreidimensionale statt sechsdimensionale Struktur hin $(\mathrm{CFI}=.97, \mathrm{TLI}=.96, \mathrm{RMSEA}=.01)$. Daher wurden alle Items mit ConQuest (Wu, Adams, Wilson \& Haldane, 2007) im Rahmen eines dreidimensionalen dichotomen Raschmodells geschätzt. Die den ursprünglichen Qualifikationsrahmen (Autorengruppe Fachschulwesen, 2011; Robert Bosch Stiftung, 2008) entnommenen Bereiche 1. Kind in Beziehung zu sich und anderen sowie 2. Kind und Welt wurden zum Faktor 1 Kindliche Bildung und Entwicklung zusammengefasst (EAP / PV-Reliabilität = .59; 10 Items). Die drei Bereiche 3. Eltern und Bezugspersonen, 4. Institution und Team sowie 5. Netzwerke wurden dem Faktor 2 Begleitende Aufgaben in der pädagogischen Arbeit und Institution zugeordnet (EAP / PV-Reliabilität $=.67 ; 20$ Items). Der ursprüngliche Bereich 6. Forschung, Recherche und Evaluation bleibt als eigenständiger Faktor bestehen, wurde jedoch in die passendere Bezeichnung 3 Forschung und Evaluation in der Kindheitspädagogik umbenannt (EAP / PV-Reliabilität = .69; 11 Items). Die Reliabilitäten können in Anbetracht der Heterogenität des erfassten Konstrukts als zumindest akzeptabel gewertet werden (Kline, 2000).

Als Richtwerte für eine passable Item-Modellpassung wurden gewichtete Abweichungsquadrate von 0.8 bis 1.2 (z.B. Bond \& Fox, 2001) gewählt, wobei ein MNSQ von 1 eine exakte Modellpassung bedeutet, zudem wurden Items mit einer Trennschärfe unter 0.2 direkt ausgeschlossen. Hierdurch ergab sich eine gekürzte Fassung des Fragebogens zur Erfassung des theoretischen Wissens mit insgesamt 41 Items. Die gewichteten Abweichungsquadrate (weighted MNSQs) der verbleibenden Items lagen zwischen 0.91 und 1.12 und sind somit durchgängig als gut zu bewerten. Bezüglich der Verteilung von Item- und Personenparametern zeigte sich eine gute Abdeckung aller Schwierigkeitsbereiche durch den FTW. Tabelle 1 fasst die Itemzuordnung, die Lösungshäufigkeiten, die gewichteten
Abweichungsquadrate sowie die Trennschärfen im Rahmen des Raschmodells zusammen.

\section{Instrumente zur Erfassung des Handlungswissens}

Bevor die psychometrische Überprüfung der Instrumente zur Erfassung des Handlungswissens erfolgte, wurde zunächst ein eigenes Auswertungsraster für beide Instrumente der Fallvignetten und Dilemmasituationen entwickelt (in Anlehnung an Fröhlich-Gildhoff et al., 2014), um der Heterogenität in der Länge der Textantworten der Teilnehmer_innen in der Onlinebefragung gerecht zu werden. Dazu wurden 200 einzelne Befragungen gesichtet und die Antworten paraphrasiert (Mayring, 2015). Anschließend wurde für jede einzelne Frage eine grobe Einteilung der Antworten in die Kategorien „korrekt“ oder „inkorrekt" vorgenommen, bevor die kategoriale Feinbestimmung in Ober- und Subkategorien erfolgte. Dem schloss sich zwecks Übertragung der Textantworten in Zahlen die Entwicklung eines Punktesystems und Codierschemas an. Im letzten Schritt wurden zur Absicherung der Entwicklung 10 komplette Onlinebefragungen ausgewertet und die Übereinstimmungen bei den Einschätzungen überprüft.

Wie beim $F T W$ wurde die Dimensionalität des Instrumentes zur Erfassung des Handlungswissens $(F H W)$ mittels Faktorenanalyse überprüft. Bei den Fallvignetten wurde erneut eine dreifaktorielle Struktur bestätigt (CFI $=.95$, $\mathrm{TLI}=.94$, RMSEA $=.033$ ). Allerdings blieben die Reliabilitäten der ermittelten Faktoren zu schwach. Lediglich übergeordnet ließ sich ein Faktor (die Güte der Handlungsideen über alle Fallvignetten hinweg) mit zufriedenstellender Reliabilität $(\alpha=.70)$ ermitteln.

Bei den Dilemmasituationen wurde entsprechend dem Vorgehen der Originalautoren der Dilemmasituationen (Fröhlich-Gildhoff et al., 2014) für die Analysen ebenfalls lediglich ein Gesamtwert Komplexitätswahrnehmung verwendet (Summen-Rohwerte aus den vier Unterfragen), welcher sich als Pendant-Wert zur Handlungsidee der Fallvignetten verhält.

\section{Gemeinsame Messung des theoretischen Konstrukts}

Zur Überprüfung der konvergenten Validität (Döring \& Bortz, 2016) wurden im letzten Schritt die Interkorrelationen zwischen den eingesetzten Verfahren berechnet.

Wurden die Ergebnisse (siehe Tab. 2) im Gesamten betrachtet, so zeigten sich in allen Fällen signifikante $\mathrm{Zu}-$ sammenhänge $(p<.001$, Spannweite $r=.19-.51$; Cohen, 
Tabelle 1. Lösungshäufigkeit der Einzelitems, $N_{\max }=831$

\begin{tabular}{|c|c|c|c|c|}
\hline Nr. U-Bereich ${ }^{1}$ & Items (Themengebiet) & Lösungshäufigkeit in \% & Weighted MNSQ & Item-Total-Correlation \\
\hline \multicolumn{5}{|c|}{ Faktor 1 „Kindliche Bildung und Entwicklung“ } \\
\hline 1 & 1. Bindungskategorien & 70.4 & 0.98 & .34 \\
\hline 1 & 2. Kindliche Emotionen & 40.9 & 1.00 & .32 \\
\hline 1 & 3. Geschlechterfragen & 56.7 & 1.01 & .29 \\
\hline 1 & 4. Theory of Mind & 22.1 & 1.03 & .22 \\
\hline 2 & 5. Gleichgewichtsregulation & 36.1 & 1.03 & .24 \\
\hline 2 & 6. Koordinative Fähigkeiten & 33.0 & 1.04 & .24 \\
\hline 2 & 7. Formen des Spiels & 52.1 & 1.02 & .28 \\
\hline 2 & 8. Sensumotorisches Spiel & 62.1 & 0.98 & .36 \\
\hline 2 & 9. Semantische Kompetenz & 32.1 & 1.03 & .22 \\
\hline 2 & 10. Mathematische Bildung & 77.6 & 0.97 & .32 \\
\hline \multicolumn{5}{|c|}{ Faktor 2 „Begleitende Aufgaben in der pädagogischen Arbeit und Institution“ } \\
\hline 3 & 11. Elternkurse & 61.7 & 0.99 & .32 \\
\hline 3 & 12. Elternarbeit & 48.0 & 1.04 & .22 \\
\hline 3 & 13. Gesprächsformate mit Eltern & 76.7 & 1.00 & .30 \\
\hline 3 & 14. Erziehungspartnerschaft & 64.1 & 1.01 & .26 \\
\hline 4 & 15. Kita-Gesetze & 38.0 & 1.02 & .27 \\
\hline 4 & 16. Kindeswohlgefährdung & 79.1 & 0.97 & .31 \\
\hline 4 & 17. Trägerarten & 67.7 & 0.94 & .43 \\
\hline 4 & 18. Gewerkschaften & 46.1 & 1.01 & .29 \\
\hline 4 & 19. Aufgaben der Kita-Leitung & 54.6 & 1.04 & .22 \\
\hline 4 & 20. Personal- und Teamentwicklung & 69.1 & 1.01 & .25 \\
\hline 4 & 21. Qualitätssicherung & 47.8 & 1.01 & .29 \\
\hline 5 & 22. Erziehungsberatung & 73.4 & 0.98 & .30 \\
\hline 5 & 23. Vernetzung & 62.6 & 0.99 & .32 \\
\hline 5 & 24. Öffentlichkeitsarbeit & 42.2 & 1.01 & .26 \\
\hline 5 & 25. Soziale Teilhabe & 71.6 & 1.00 & .28 \\
\hline 5 & 26. Zusammenarbeit mit Schulen & 28.4 & 1.01 & .27 \\
\hline 5 & 27. Sozialpädiatrische Zentren & 43.4 & 1.04 & .21 \\
\hline 5 & 28. Integrationsfachkraft & 52.2 & 0.98 & .32 \\
\hline 5 & 29. Gesundheitsprogramme & 33.7 & 0.98 & .34 \\
\hline 5 & 30. zahnärztliche Untersuchung & 59.2 & 1.03 & .22 \\
\hline \multicolumn{5}{|c|}{ Faktor 3 „Forschung und Evaluation in der Kindheitspädagogik“ } \\
\hline 6 & 31. Wissenschaftliche Artikel & 40.8 & 1.10 & .31 \\
\hline 6 & 32. Messgenauigkeit & 70.3 & 0.97 & .42 \\
\hline 6 & 33. Beobachtungen im Kita-Alltag & 61.7 & 1.01 & .36 \\
\hline 6 & 34. Führen von Interviews & 72.1 & 0.96 & .38 \\
\hline 6 & 35. Standardisierte Testverfahren & 49.1 & 0.96 & .47 \\
\hline 6 & 36. Durchführung von Beobachtungen & 32.3 & 1.03 & .37 \\
\hline 6 & 37. Eigenschaften von Beobachtungen & 40.0 & 0.91 & .54 \\
\hline 6 & 38. Umgang mit Daten & 53.8 & 1.03 & .37 \\
\hline 6 & 39. Objektive Beobachtungen & 34.1 & 1.12 & .28 \\
\hline 6 & 40. Qualitätskriterien von Testverfahren & 43.3 & 1.10 & .33 \\
\hline 6 & 41. Externe Beurteilungen & 53.5 & 1.01 & .40 \\
\hline
\end{tabular}

Anmerkungen: ${ }^{1}$ Ursprüngliche Bereiche nach dem Qualifikationsrahmen. 
Tabelle 2. Interkorrelation der eingesetzten Verfahren nach Pearson $\left(N_{F T W}=831, N_{F T W / F H W-D H W}=418\right)$

\begin{tabular}{|c|c|c|c|c|c|}
\hline & FTW Faktor 1 & FTW Faktor 2 & FTW Faktor 3 & FHW HI & $D H W K W$ \\
\hline FTWa Faktor 1 & - & $.366 *$ & $.356 *$ & $.464^{\star}$ & $.230 *$ \\
\hline FTW Faktor 2 & $.366^{*}$ & - & $.507 *$ & $.398 *$ & $.267 *$ \\
\hline FTW Faktor 3 & $.356^{\star}$ & $.507 \star$ & - & $.329 *$ & $.185^{\star}$ \\
\hline FHW HI & $.464^{\star}$ & $.398 *$ & $.329 *$ & - & $.460 *$ \\
\hline DHW KW & $.230 *$ & $.267 *$ & $.185^{\star}$ & $.460 *$ & - \\
\hline
\end{tabular}

Anmerkungen: * $p<.001 .{ }^{b}$ Gesamtwert „Handlungsidee“ der Fallvignetten. ${ }^{\circ}$ Gesamtwert „Komplexitätswahrnehmung“ der Dilemmasituationen

1988), wobei die Stärke der einzelnen Korrelationen erwartungskonform war (mittlere bis hohe Korrelationen zwischen den einzelnen Faktoren des FTWs sowie zum übergreifenden Konstrukt Handlungsidee - eher geringe Korrelationen zwischen dem FTW und der Komplexitätswahrnehmung).

\section{Diskussion}

Insgesamt zeigen die Ergebnisse erste Möglichkeiten auf, Facetten kindheitspädagogischer Fachkompetenz - Theoretisches Wissen und Handlungsbezogenes Wissen - von angehenden und ausgebildeten pädagogischen Fachkräften mit den entwickelten und psychometrisch überprüften Instrumenten zu erfassen. Allerdings ergaben sich Differenzen zu den Voraussetzungen der ausgewählten Grundlagenmodelle: Statt der in den Orientierungsrahmen für Hoch- und Fachschulen (Autorengruppe Fachschulwesen, 2011; Robert Bosch Stiftung, 2008) verankerten sechs Kompetenzbereiche konnte beim FTW eine dreidimensionale Struktur festgestellt werden. Auch die Überprüfung der beiden Instrumente, die noch deutlicher auf dem Kompetenzmodell von Fröhlich-Gildhoff et al. (2011, 2014) basieren und stärker das Handlungswissen fokussieren, weist eher auf eine übergreifende Strukturierung hin: Bei den Fallvignetten ergab sich zunächst eine drei- statt sechsdimensionale Struktur. Aufgrund zu schwacher Reliabilitäten, die auf die geringe Anzahl an Unterfragen oder auch teilweise deren Beschaffenheit bei diesem Erhebungsinstrument zurückzuführen sind, wies das Modell mit einem übergeordneten Faktor (Handlungsidee) die beste Modellgüte auf. Auch bei den Dilemmasituationen erwies sich - allerdings in Entsprechung des Modells von Fröhlich-Gildhoff et al. (2014) - ein übergeordneter Faktor (Komplexitätswahrnehmung) als beste Modellierung.

Die Reduktion bzw. Zusammenführung mehrerer Einzelfaktoren der Fachkompetenz auf einen oder wenige übergeordnete Faktoren bzw. Facetten lässt sich einbetten in den wissenschaftlichen Diskurs zu diesem Thema. So gehen Seifert und Schaper (2010) in ihrem Kompetenzmodell im Rahmen der Lehrer_innenbildung eher von einem Generalfaktor der Fachkompetenz aus. In ihren Untersuchungen zur Entwicklung mathematischer Lehr(er_innen)kompetenz von Blömeke und König (2010) zeigte sich hingegen, dass ein Modell der „Heterogenität der pädagogischen Subdimensionen " (Blömeke \& König, 2010, S.263) „Strukturierung von Unterricht“, „Umgang mit Heterogenität“, „Klassenführung und Motivation“ sowie „Leistungsbeurteilung" eine tendenziell bessere Güte gegenüber dem Generalfaktor „Pädagogisches Wissen“ hatte. Die Überlegenheit des heterogenen Modells zeigte sich deutlicher bei Lehrer_innen in Ausbildung; eine stärkere Homogenität der Einzelfaktoren war bei berufserfahrenen Lehrkräften zu finden.

Die (bisher wenigen) Untersuchungen bei pädagogischen Fachkräften im Feld der frühkindlichen Bildung, Betreuung und Erziehung sprechen gleichfalls für ein Modell einer eher homogenen Struktur der Fachkompetenz mit zwei Faktoren (Strohmer \& Mischo, 2014; 2016). Auch Granzow (2017, S.150) folgert im Fazit seiner Untersuchung: ,Berufliche Kompetenz umfasst [...] Kompetenzen spezifisch-inhaltlicher Anforderungen, die ein Bündel von Fähigkeiten und Fertigkeiten darstellen (berufliches Handlungswissen und Können), um spezifische Anforderungsbereiche des Arbeitskontextes zu bewältigen (Umsetzung/Können)". Damit lässt sich folgern, dass bezüglich des Theoriewissens eine konzeptuelle Unterscheidung nach verschiedenen, wenigen Kompetenzaspekten bzw. -bereichen der Kindheitspädagogik möglich ist; das Handlungswissen - in der bestätigten Konzipierung als Handlungsidee und Komplexitätswahrnehmung - in diesem Fall hingegen jedoch eher gesamtheitlich betrachtet werden sollte. Bei der Betrachtung überfachlicher Kompetenzfacetten könnte sich möglicherweise eine heterogenere Struktur ergeben, wie die Untersuchung von Verbeek und Perels (2018) zeigte. Dieser Frage sollte weiter nachgegangen werden.

Mittels Berechnung der Interkorrelation konnte die konvergente Validität zwischen den eingesetzten Verfahren weitgehend überprüft werden. Die eher niedrigen $\mathrm{Zu}-$ sammenhänge zur Komplexitätswahrnehmung in uneindeutigen Situationen können neben der grundsätzlichen 
Unterschiedlichkeit der erforderlichen Denkweise (hier geht es nicht um richtig oder falsch, sondern vielmehr um flexible Mehrperspektivität) auch auf inhaltliche Aspekte zurückgeführt werden. Des Weiteren kann hier ein entwicklungsbedingtes Phänomen zugrunde liegen, da die Dilemmasituationen nur zu den Kompetenzbereichen 1-4 entwickelt wurden. So liegt die niedrigste Korrelation $(r=$ .20) zum 3. Faktor des $F T W$ vor, dessen Inhalt „Forschung und Evaluation in der Kindheitspädagogik" bei den Dilemmasituationen somit nicht erfragt wurde.

Insgesamt betrachtet ist die Messgenauigkeit der Instrumente zumindest akzeptabel. So zeigte sich unter anderem eine mittlere Itemschwierigkeit bei den Items und Faktoren des FTWs sowie ein insgesamt breiteres Spektrum sowohl an leichteren, als auch schwereren Aufgaben, sodass in einem weiteren Schritt ggf. auch im Sinne eines Kompetenzstufenmodells (Klieme, Maag Merki \& Hartig, 2007) erste Unterscheidungen von Kompetenzniveaus angedacht werden können. Bei den Instrumenten zur Erfassung des Handlungswissens konnte zudem ein überprüftes Punkte- und Codierschemata entwickelt werden, welches es ermöglicht, Textantworten der Teilnehmer_innen standardisiert auszuwerten.

Zusammengefasst konnte mit der Entwicklung der drei Erhebungsinstrumente ein erster Schritt hinsichtlich der Verringerung des Desiderats zu den Möglichkeiten der Messung von Facetten der Fachkompetenz früh- bzw. kindheitspädagogischer Fachkräfte geleistet werden. Inwiefern die Instrumente hilfreich sind, um als Methode der Lern- und Professionalisierungsbegleitung in der Ausund Weiterbildung zu fungieren, muss sich in den nachfolgenden Analysen (Lorenzen, Limberger, Wirth, Strohmer \& Fröhlich-Gildhoff, 2020) des Projekts zeigen, in dessen Rahmen die hier beschriebene Entwicklung und Überprüfung der Instrumente erfolgt ist.

\section{Literatur}

Autorengruppe Fachschulwesen (2011). Qualifikationsprofil „Frühpädagogik" - Fachschule/Fachakademie (Bd. 1). München. Verfügbar unter: https://www.weiterbildungsinitiative.de/uploads/ media/WiFF_Kooperationen_1_Qualifikationsprofil_Internet. pdf

Barter, C. \& Renold, E. (1999). The use of vignettes in qualitative research. Social research update, 25. Verfügbar unter: https://sru. soc.surrey.ac.uk/SRU25.html

Blömeke, S., Jenßen, L., Dunekacke, S., Suhl, U., Grassmann, M. \& Wedekind, H. (2015). Leistungstests zur Messung der professionellen Kompetenz frühpädagogischer Fachkräfte. Zeitschrift für pädagogische Psychologie, 29, 177-191. https://doi.org/ 10.1024/1010-0652/a000159

Blömeke, S. \& König, J. (2010). Messung des pädagogischen Wissens: Theoretischer Rahmen und Teststruktur. In S. Blömeke, G. Kaiser \& R. Lehmann (Hrsg.), TEDS-M 2008. Professionelle Kom- petenz und Lerngelegenheiten angehender Mathematiklehrkräfte für die Sekundarstufe I im internationalen Vergleich (S. 239 -263). Münster: Waxmann.

Bond, T. G. \& Fox, C. M. (2001). Applying the Rasch Model: Fundamental Measurement in the Human Sciences. New York, NY: Lawrence Erlbaum. https://doi.org/10.4324/9781410600127

Bundesministerium für Bildung und Forschung (2020). Kompetenzmodellierung und Instrumente der Kompetenzerfassung im Hochschulsektor. Verfügbar unter: https://www.wihoforschung. de/de/kompetenzmodellierung-und-kompetenzerfassung605.php.

Cohen, J. (1988). Statistical power analysis for the behavioral sciences. Hillsdale, MI: Erlbaum.

Döring, N. \& Bortz, J. (2016). Forschungsmethoden und Evaluation in den Sozial- und Humanwissenschaften (5., vollst. überarb., aktual. und erw. Aufl.). Berlin: Springer. https://doi.org/10.1007/ 978-3-642-41089-5

Drieschner, E. (2009). Bildungsstandards praktisch. Wiesbaden: VS Verlag für Sozialwissenschaften. https://doi.org/10.1007/9783-531-91343-8

Frey, A. (2006). Methoden und Instrumente zur Diagnose beruflicher Kompetenzen von Lehrkräften. Eine erste Standortbestimmung zu bereits publizierten Instrumenten. In C. Allemann-Ghionda \& E. Terhart (Hrsg.), Kompetenzen und Kompetenzentwicklung von Lehrerinnen und Lehrern (S.30 - 46). Weinheim: Beltz.

Fröhlich-Gildhoff, K. \& Böttinger, U. (Hrsg.) (2018). Prävention und Gesundheitsförderung als kommunale Gesamtstrategie. Konzept, Entwicklung und Evaluation des Präventionsnetzwerks Ortenaukreis (PNO). Freiburg: FEL.

Fröhlich-Gildhoff, K., Pietsch, S., Wünsche, M. \& Rönnau-Böse, M. (Hrsg.) (2011). Zusammenarbeit mit Eltern in Kindertageseinrichtungen. Ein Curriculum für die Aus- und Weiterbildung. Materialien zur Frühpädagogik. Band 7. Freiburg: FEL.

Fröhlich-Gildhoff, K., Nentwig-Gesemann, I., Pietsch, S., Köhler, L. \& Koch, M. (2014). Kompetenzentwicklung und Kompetenzerfassung in der Frühpädagogik. Konzepte und Methoden. Materialien zur Frühpädagogik. Band 13. Freiburg: FEL.

Fröhlich-Gildhoff, K., Rönnau-Böse, M. \& Tinius, C. (2017). Herausforderndes Verhalten in Kita und Grundschule. Erkennen, Verstehen, Begegnen. Stuttgart: Kohlhammer.

Granzow, M. (2017). Berufliche Kompetenzen frühpädagogischer Fachkräfte. Entwicklung eines Struktur- und Messmodells zur Erfassung beruflicher Kompetenzen im Praxisfeld der Kindertagesstätte. Freie Universität Berlin.

Hohenstein, F., Kleickmann, T., Zimmermann, F., Köller, O. \& Möller, J. (2017). Erfassung von pädagogischem und psychologischem Wissen in der Lehramtsausbildung: Entwicklung eines Messinstruments. Zeitschrift für Pädagogik, 63(1), 91-113.

Hunkirchen, P. (2005). Bitte laut denken: „Thinking Aloud“. Verfügbar unter: http://www.fit-fuer-usability.de/archiv/bitte-lautdenken-thinking-aloud/

Klaudy, E. K., Schütz, A. \& Stöbe-Blossey, S. (2014). Akademisierung der Ausbildung für die Kindertageseinrichtung. Zur Entwicklung kindheitspädagogischer Studiengänge. Institut Arbeit und Qualifikation an der Universität Duisburg-Essen. IAQ-Report, Nr. 2014-04. Verfügbar unter: http://www.iaq.uni-due.de/ iaq-report/2014/report2014-04.pdf

Kline, P. (2000). The Handbook of Psychological Testing. London: Routledge.

Klieme, E., Maag Merki, K. \& Hartig, J. (2007). Kompetenzbegriff und Bedeutung von Kompetenzen im Bildungswesen. In J. Hartig \& E. Klieme (Hrsg.), Möglichkeiten und Voraussetzungen technologiebasierter Kompetenzdiagnostik (S.1-15). Bonn: Bundesministerium für Bildung und Forschung.

Kuckarz, D., Mackowiak, K., Ziroli, S., Kauertz, A., RathgebSchnierer, E. \& Dieck, M. (Hrsg.). (2014). Professionelles Han- 
deln im Elementarbereich (PRIMEL). Eine deutsch-schweizerische Videostudie. Münster: Waxmann.

Konrad, K. (2010). Lautes Denken. In G. Mey \& K. Mruck (Hrsg.), Handbuch Qualitative Forschung in der Psychologie (S.476 - 490). Wiesbaden: VS Verlag für Sozialwissenschaften. https://doi.org/10.1007/978-3-531-92052-8_34

Lorenzen, A., Limberger, J., Wirth, C., Strohmer, J. \& FröhlichGildhoff, K. (2020). Individuelle kompetenzorientierte Feedbacks als Methode der Professionalisierungsbegleitung frühpädagogischer Fachkräfte (Projekt InKoFeed). Wissenschaftlicher Abschlussbericht. Freiburg: FEL.

Mayring, P. (2015). Qualitative Inhaltsanalyse: Grundlagen und Techniken (12. Aufl.). Weinheim: Beltz.

Muthén, L. K. \& Muthén, B. (2010). Mplus. Statistical Analysis With Latent Variables. User's Guide. Los Angeles, CA: Muthén \& Muthén.

Mischo, C. \& Fröhlich-Gildhoff, K. (2011). Professionalisierung und Professionsentwicklung im Bereich der frühen Bildung. Frühe Bildung, 0, 4-12. https://doi.org/10.1026/2191-9186/ a000001

Mischo, C., Wahl, S., Hendler, J., Maack, M., Strohmer, J. \& Wolf, C. (2016). Schlussbericht des Projekts Ausbildung und Verlauf von Erzieherinnen-Merkmalen (AVE). Verfügbar unter: https://www. phfreiburg.de/fileadmin/dateien/fakultaet1/psychologie/ Uploads/Mischo/AVE_Abschlussbericht.pdf

Mischo, C. (2017). Information zu zentralen Ergebnissen des Projekts „Ausbildung und Verlauf von Erzieherinnen-Merkmalen (AVE)“. Frühe Bildung, 6, 172-175. https://doi.org/10.1026/ 2191-9186/a000339

Neuweg, G. H. (2015). Kontextualisierte Kompetenzmessung. Eine Bilanz zu aktuellen Konzeptionen und forschungsmethodischen Zugängen. Zeitschrift für Pädagogik, 61, 377 - 383.

Robert Bosch Stiftung (2008). Frühpädagogik studieren - ein Orientierungsrahmen für Hochschulen. Verfügbar unter: http:// www.bosch-stiftung.de/content/language1/downloads/PiK_ orientierungsrahmen_druckversion.pdf

Seifert, A. \& Schaper, N. (2010). Überprüfung eines Kompetenzmodells und Messinstruments zur Strukturierung allgemeiner pädagogischer Kompetenz in der universitären Lehrerbildung. Lehrerbildung auf dem Prüfstand, 3,179-198.

Strohmer, J. \& Mischo, C. (2014). Aufgaben und Funktionen von Kindertageseinrichtungen aus Sicht angehender frühpädagogischer Fachkräfte - welche Rolle spielen Ausbildungsart und Ausbildungszeitpunkt? In K. Fröhlich-Gildhoff, I. Nentwig-Gesemann \& N. Neuß (Hrsg.), Forschung in der Frühpädagogik. 7. Schwerpunkt:Profession und Professionalisierung (S.173 - 196). Freiburg: FEL.
Strohmer, J. \& Mischo, C. (2016). Does early childhood teacher education foster professional competencies? The development of professional competencies in different education tracks in Germany. Early child development and care, 186(1), 42 - 60. https:// doi.org/10.1080/03004430.2014.985217

Verbeek, V. (2016). Modellierung, Messung und Analyse berufsrelevanter überfachlicher Kompetenzen in der fachschulischen Erzieherausbildung. Universität des Saarlandes: Saarbrücken.

Verbeek, V. \& Perels, F. (2018). Modellierung und Validierung überfachlicher Kompetenzen angehender ErzieherInnen und Erzieher. Empirische Pädagogik, 32(1), $63-76$.

Weinert, F. E. (2001). Concept of competences: A conceptual clarification. In D. S. Rychen \& L. H. Salgnik (Eds.), Defining and Selecting Key Competencies. Theoretical and Conceptual Foundations (pp. 45 -65). Cambridge, MA: Hogrefe Publishing.

Weltzien, D. \& Lorenzen, A. (2016). Kinder Stärken! Förderung von Resilienz und seelischer Gesundheit in Kindertageseinrichtungen. Wissenschaftlicher Abschlussbericht. Freiburg: FEL.

Wittek, D., Schneider E. \& Kramer R.-T. (2020). Strukturtheoretischer Professionsansatz und Evidenzbasierung? Ein neues Instrument zur Erfassung professionalisierungsrelevanter fachübergreifender Merkmale von Lehramtsstudierenden. In I. Gogolin, B. Hannover \& A. Scheunpflug (Hrsg.), Evidenzbasierung in der Lehrkräftebildung (S. 265 - 289). Wiesbaden: Springer. https://doi.org/10.1007/978-3-658-22460-8_11

Wu, M. L., Adams, R. J., Wilson, M. R. \& Haldane, S. A. (2007). ACER ConQuest 2.0: Generalised Item Response Modelling Software. Camberwell: Australian Council for Educational Research Ltd.

\section{Autorenschaften}

Die Mitautor_innen sind erreichbar unter: Annika Lorenzen, annika. lorenzen@eh-freiburg.de; Charlotta Wirth, charlotta.wirth@ehfreiburg.de; Janina Strohmer, janina.strohmer@ph-freiburg.de; Klaus Fröhlich-Gildhoff, klaus.froehlich-gildhoff@eh-freiburg.de

\section{Förderung}

Open Access-Veröffentlichung ermöglicht durch Evangelische Hochschule Freiburg.

\section{Janina Limberger}

Zentrum für Kinder- und Jugendforschung (ZfKJ)

an der Evangelischen Hochschule Freiburg

Bugginger Str. 38

79114 Freiburg

janina.limberger@eh-freiburg.de 